\title{
Micrometastasis of endometriosis to distant organs in a murine model
}

\author{
Elham N. Samani ${ }^{1}$, Ramanaiah Mamillapalli' ${ }^{1}$ Fei Li $^{1}$, Levent Mutlu ${ }^{1}$, Demetra \\ Hufnagel $^{1}$, Graciela Krikun ${ }^{1}$ and Hugh S. Taylor ${ }^{1}$ \\ ${ }^{1}$ Department of Obstetrics, Gynecology and Reproductive Sciences, Yale School of Medicine, Connecticut 06510, New Haven, USA \\ Correspondence to: Ramanaiah Mamillapalli, email: ramana.mamillapalli@yale.edu \\ Keywords: endometriosis, micrometastasis, DsRed cells, metastasis, mice
}

Received: January 13, 2017 Accepted: March 30, 2017 Epub: April 06, 2017 Published: March 19, 2019

Copyright: Samani et al. This is an open-access article distributed under the terms of the Creative Commons Attribution License 3.0 (CC BY 3.0), which permits unrestricted use, distribution, and reproduction in any medium, provided the original author and source are credited.

\section{ABSTRACT}

Endometriosis is an inflammatory gynecological disorder among reproductiveaged women caused by the engraftment and proliferation of endometrial cells outside the uterus, most commonly in the pelvis. It is thought that the disease arises primarily from retrograde menstruation where cells from the endometrium travel through the fallopian tubes to the peritoneal cavity. However, migration of endometriosis-derived cells to distant organs outside of the peritoneal cavity have not been explored. In the present study, we developed and validated a mouse model of disseminated endometriosis using syngeneic DsRed endometrial tissue introduced into the peritoneum of immunocompetent mice. Flow cytometry and immunofluorescence analysis, demonstrated the presence of endometriosis-derived cells in multiple organs (including lung, spleen, liver and brain) in the murine endometriosis model. Immunostaining revealed the presence of DsRed $^{+} / \mathrm{CD}^{-} 5^{-}$cells in brain, liver and lung. Engraftment occurred in all experimental animals examined. Cells from endometriotic lesions are capable of migration to and engraftment of multiple organs outside of the peritoneal cavity. Micrometastasis of endometriosis is a novel and frequent phenomenon. These data suggest that widespread dissemination of endometriosis may be common, clinically unrecognized and contribute to the diffuse clinical manifestations of this disease.

\section{INTRODUCTION}

Endometriosis is a common gynecological disorder among reproductive aged women (6-10\%) [1] due to the deposition and growth of endometrial cells outside the uterus $[2,3]$. Approximately, $50 \%$ of patients with endometriosis have severe pelvic pain and $40-50 \%$ have infertility $[4,5]$ negatively affecting the health and quality of life of these patients $[1,6]$. Even though, the diagnosis of superficial or subtle lesions in endometriosis is still challenging, imaging modalities have increased sensibility and specificity for detecting endometriosis in the abdomen. But the true incidence in the general population is unknown.

Despite being one of the most common gynecologic diseases, the pathogenesis of endometriosis is poorly understood $[7,8]$. The most widely accepted theory of its etiology is ectopic implantation, or Sampson's theory, which proposes that endometrial cells are shed out of the uterus through retrograde menstruation, thereby gaining access to and implanting on pelvic structures [9]. However, implants of endometriosis outside of the peritoneal cavity, in women affected by Mayer-Rokitansky-Kuster-Hauser's syndrome (absent uterus) or reports of endometriosis in men cannot be explained by these theories $[10,11]$. The theory of vascular dissemination suggests that endometrial cells may enter the uterine vasculature or lymphatic system at menstruation and are transported to other sites [12]. Finally, our group has described a stem cell origin of endometriosis; endometriosis arises from ectopic differentiation of circulating mesenchymal stem cells [7, 13-16].

Endometriosis is most commonly found in the pelvis. However, it is also found in organs not contiguous with the peritoneal cavity; these include the pericardium, lung, brain, and skin $[3,7,8,17-19]$. One review of pathology specimen reports revealed that endometriosis has been identified in nearly all human organs [20]. The exact 
incidence and pathogenesis of extra pelvic endometriosis is unknown due to the presumed relative rarity of this condition, the lack of effective imaging, and the regression of disease after menopause [17, 21, 22]. It is typically identified at these atypical sites when patients present with atypical, cyclic, clinical symptoms. Women with pleural lesions, in particular, may experience menstrual pneumothorax or hemoptysis [23]. Central nervous system involvement has presented as catamenial headaches, seizures or subarachnoid hemorrhage [17, 24]. These data suggest that dissemination of endometriosis may be widespread.

In this study, we used a murine model of surgically induced endometriosis to determine whether endometriosis-derived cells are capable of migration or micrometastases to distant organs, including the lung, spleen, liver, or brain. Micrometastasis is a form of metastasis where the spread and engraftment of a small number of cells shed from a primary location to another part of the body are too minuscule to be detected by the conventional detection methods [25]. Previously we have identified cell migration from endometriosis within the pelvic cavity to the uterus [26]. We extend those findings and identified the frequent migration of endometriosisderived cells to extra pelvic organs, suggesting that micrometastasis of endometriosis may be a common phenomenon.

\section{RESULTS}

\section{Migration of DsRed ${ }^{+}$cells from experimental endometriosis to lung, spleen, liver, and brain}

To determine whether endometriosis lesions have cells with the ability to migrate from experimental endometriosis to the lung, we performed Fluorescence Activated Cell Sorting (FACS). As expected, DsRed ${ }^{+}$cells were found in the lungs of all DsRed ${ }^{+}$positive control mice. No DsRed ${ }^{+}$cells were observed in the lungs of the negative controls that underwent sham surgery. Small numbers of DsRed ${ }^{+}$cells were identified in the lungs of all animals at eight weeks after induction of endometriosis. Experiments were carried out 3 times with different samples and each time in duplicate. (Figure 1). The mean percentage of DsRed ${ }^{+}$cells in the lungs of animals with endometriosis was $0.0028 \pm 0.0003$ (Figure 2).

The DsRed ${ }^{+}$cell population from the spleen in each group (DsRed ${ }^{+}$positive control, sham negative control and endometriosis model) were evaluated using FACS (Figure 1). An average of $0.0017 \% \pm 0.0004$ of the total cells in the spleens of animals with endometriosis were DsRed ${ }^{+}$(Figure 2). No DsRed ${ }^{+}$cells were observed in the spleens of the sham controls. Positive controls demonstrated exclusively DsRed ${ }^{+}$cells (Figure 1). Similarly, analysis of liver cells by FACS revealed an average of $0.0074 \% \pm 0.0015$ DsRed $^{+}$cells in the livers of the mice with experimental endometriosis (Figure 2). Finally, to identify whether endometriosis-derived cells are capable of migration to the brain, the brain cells were subjected FACS as shown in Figure 1. On average $0.0043 \% \pm 0.0010$ of the total cells in the brain expressed DsRed $^{+}$fluorescence in the endometriosis induced mice (Figure 2). The number of DsRed ${ }^{+}$cells differed in each organ; DsRed ${ }^{+}$cells were found at the highest frequency in the liver and at the lowest frequency in the spleen. A significantly higher percentage of $\mathrm{DsRed}^{+}$cells was observed in the liver compared to the lung, brain $(p=0.01)$ and spleen $(p=0.001)$ in the endometriosis model (Figure 2).

\section{Localization of the endometriosis derived cells in each organ}

We then sought to confirm the presence of DsRed ${ }^{+}$ cells migrating from endometriosis to the multiple extra pelvic organs. Therefore, we examined paraffin-embedded tissue sections using immunostaining. Lung, liver and brain sections of all animals were visualized under a fluorescence microscope in two independent experiments. Figure 3A displays representative confocal micrographs of lung parenchyma from our three different groups consisting of the DsRed (positive control), sham surgery (negative control) and endometriosis-induced mice. Lung tissue from DsRed ${ }^{+}$mice was highly fluorescent and demonstrated exclusively DsRed expressing cells as shown in Figure 3A. Mice that had undergone sham surgery displayed no DsRed fluorescence and DsRed expressing cells were observed in the lung tissue of mice with experimental endometriosis. Moreover, DsRed ${ }^{+}$cells were most frequently observed in the bronchial compartment of lungs of endometriosis mice. CD45, a pan leukocyte marker, was used to identify leukocytes within the lesions. The DsRed ${ }^{+}$cells identified in the lungs of mice with endometriosis did not express CD45. This suggests that the migratory cells were not leukocytes. As expected, no $\operatorname{DsRed}^{+}$cells were observed in the sham model, while $\mathrm{CD} 45^{+}$cells were observed (Figure $3 \mathrm{~B}$ ). High power view of the lung tissue demonstrating CD45/DsRed ${ }^{+}$cells in the lung are shown in Figure $4 \mathrm{~A}$ and $4 \mathrm{~B}$, respectively. In the same way, we examined whether endometriosis-derived cells were capable of migration to the liver in the mice with endometriosis. After immunofluorescent labeling, DsRed ${ }^{+}$ cells were found to localize in the liver parenchyma (Figure 4A). We found no DsRed ${ }^{+}$cells in sham model livers. Then, we performed double staining for DsRed and CD45 on all three groups. The DsRed ${ }^{+}$cells did not express CD45 in the endometriosis mice. These findings were similar to those of the other organs, indicating the nonhematopoietic origin of the endometriosis-derived DsRed $^{+}$cells (Figure 4B). Finally, the presence of DsRed $^{+}$cells in the cerebral tissue of the endometriosis model mice was analyzed using immunofluorescent 
staining. No DsRed expression was observed in sham model mice, whereas the DsRed positive control tissue sections demonstrated expected strong DsRed positivity (Figure 4A). Notably, in the recipient mice, the migrated DsRed $^{+}$cells in the brain appeared predominantly in the hypothalamus, hippocampus, and superficial layer of the cerebral cortex. DsRed ${ }^{+}$cells were negative for CD45 in the mice with experimental endometriosis (Figure 4B). These findings again support the nonhematopoietic origin of the endometriosis-derived DsRed ${ }^{+}$cells.
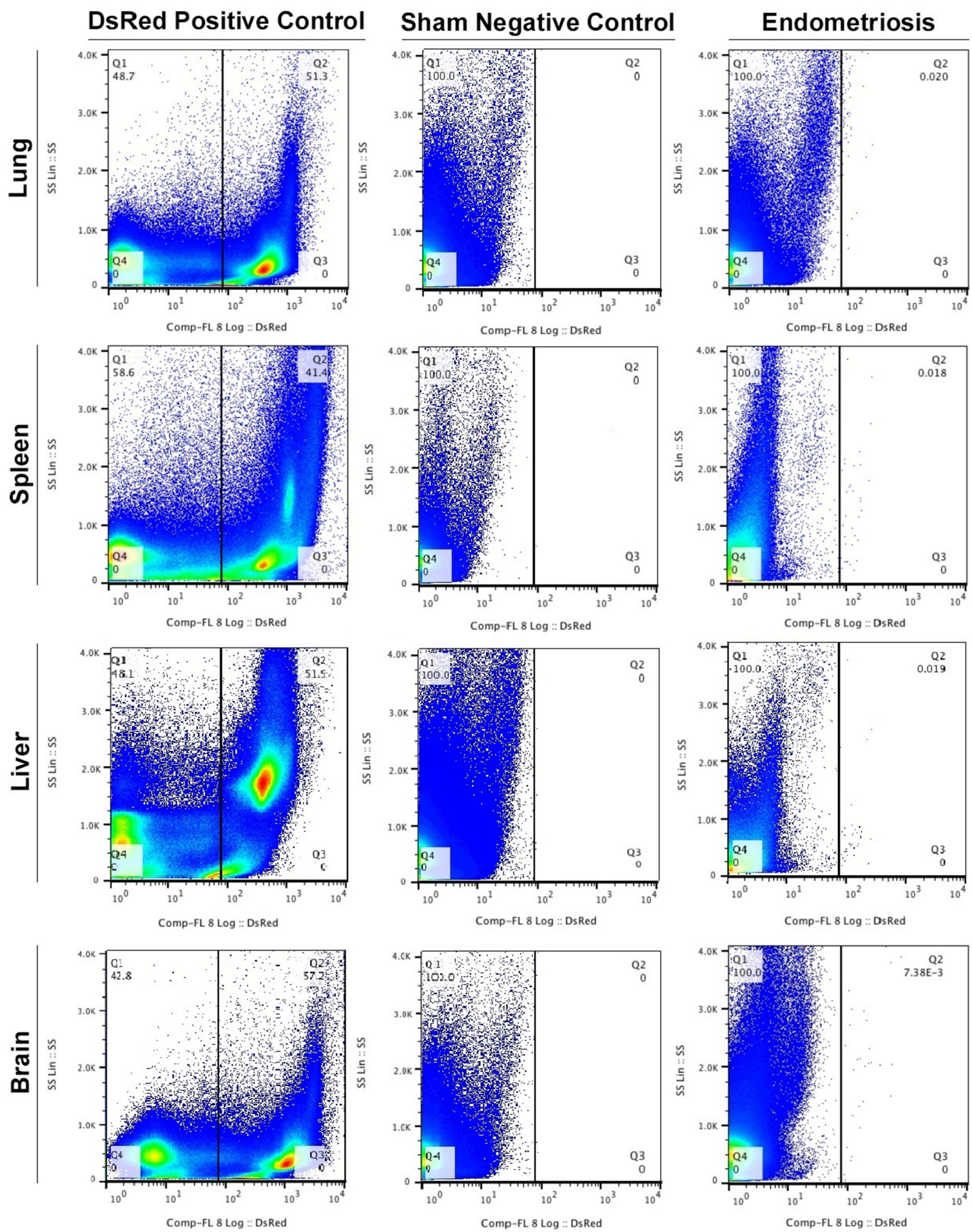

Figure 1: FACS profiles of the DsRed ${ }^{+}$endometriosis-derived cells in extrapelvic organs. Fluorescence Activated Cell Sorting (FACS) was performed to identify DsRed ${ }^{+}$cells in all animals. (Left to right): Transgenic DsRed mouse (positive control), Sham surgery (negative control) and the experimental endometriosis model. Experiments were performed 3 times with different samples and each time in duplicate. Results (\% of cells) presented are the average of triplicates. 


\section{DISCUSSION}

We demonstrate here that endometriosis-derived cells are capable of migration to extrapelvic organs including the lung, spleen, liver and brain. The presence of endometriosis-derived cells in all of the endometriosis mice tested suggests that migration of endometriosis to organs outside of the pelvis is common. Microscopic implants of endometriosis may be biologically functional and may explain some of the systemic manifestations of endometriosis in humans. As endometriosis is associated with various systemic abnormalities in humans, the relatively high recruitment of cells from the transplanted endometrial tissue to the brain and liver is particularly interesting.

Endometriosis is generally considered to be a reproductive tract disease, causing pelvic pain and infertility [27]. However women with endometriosis often experience a more broad spectrum of debilitating symptoms including non-pelvic pain, fatigue, malaise, eating disorders, anthropometric variation, endocrine and metabolic dysfunction, immunologic defects, and sociopsychological issues that impair their quality of life [28-33]. The underlying mechanisms that produce this wide array of symptoms that transcend the pelvis are unknown [34]. We speculate that some may be due to undiagnosed cellular infiltration with endometriosis. The most common sites of endometriosis in humans are the ovaries, anterior and posterior uterine culs-de-sac, posterior broad ligaments, uterosacral ligaments, uterus, fallopian tubes, sigmoid colon, appendix, and uterine round ligaments [35]. While extrapelvic endometriosis is thought to be rare, it has been found in the gastrointestinal tract $(32.3 \%)$, urinary tract $(5.9 \%)$, and distant sites including the lung, umbilicus, skin scars, liver, gallbladder, pancreas, breast, and extremities [36, 37]. Involvement of the vertebrae, bone, peripheral nerves, diaphragm, spleen, and central nervous system have also been reported [38]. Here, we describe the common and uniform occurrence of micrometastasis of endometriosis to multiple organs.

We demonstrated the presence of endometriosisderived cells in the bronchial compartment in a murine model. Thoracic endometriosis is the most common extrapelvic location in humans and primates [37, 39]. Thoracic endometriosis can involve the pleura, parenchyma, diaphragm, and bronchi, which may present as catamenial pneumothorax, hemothorax, hemoptysis, chest pain, or dyspnea [39, 40]. Intrathoracic endometriosis lesions probably go unrecognized due to inaccessibility of these lesions at imaging or bronchoscopy [39, 41]. More subtle variants may exist that result in symptoms that are ignored or dismissed.

Clinically, endometriosis within the liver has been reported; however, it is rarely recognized $[42,43]$. Hepatic endometriosis has resulted in jaundice and portal vein thrombosis [43]. Unusual symptoms such as refractory right shoulder pain have additionally been a presenting symptom [44]. Hepatic endometriosis has appeared as solitary nodules or as multiple intra/ extra hepatic cystic lesions on imaging [45]. We observed a relatively high recruitment of cells from the transplanted endometrial tissue to the liver, which is

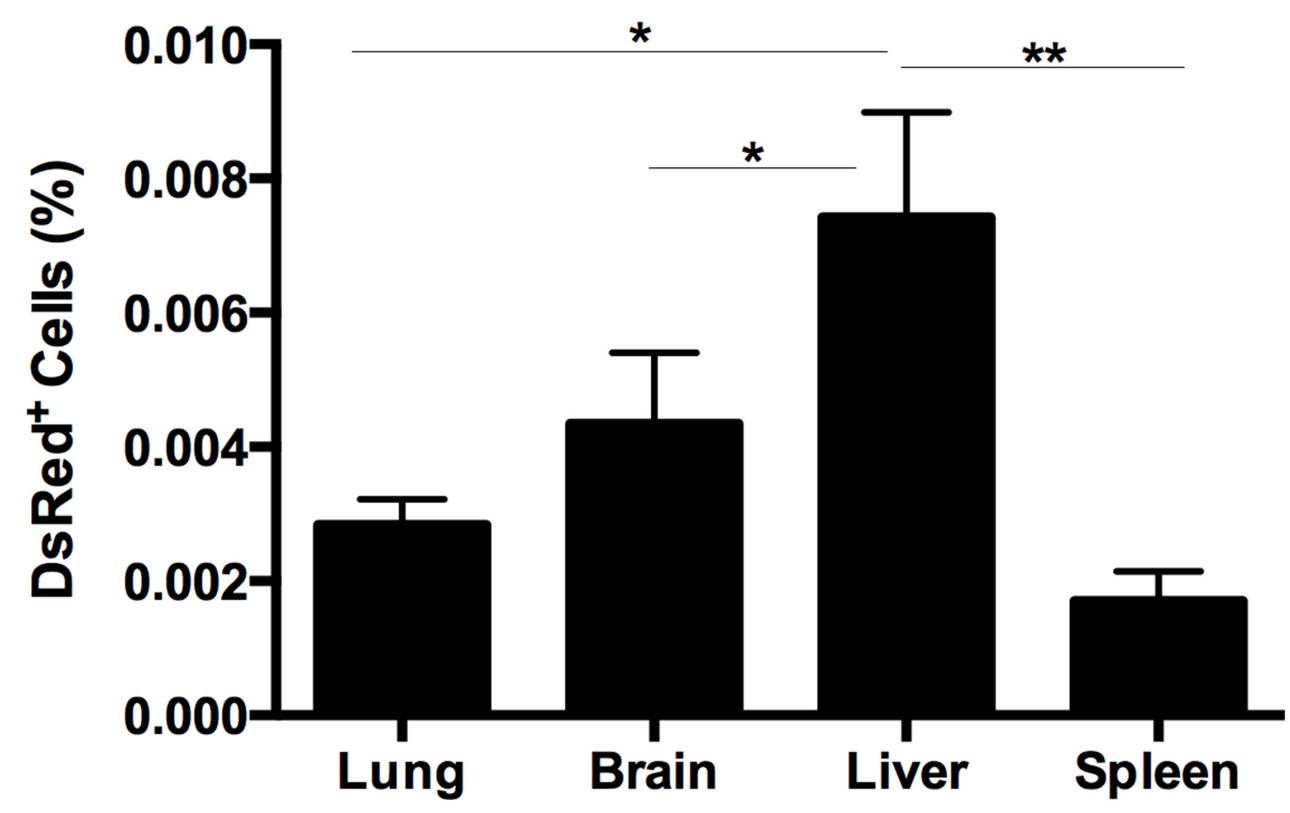

Figure 2: Quantitative analysis of the DsRed expression in lung, spleen, liver, and brain in endometriosis mice. FACS analysis was used to quantify the number of DsRed ${ }^{+}$cells. The number of DsRed, i.e. endometriosis derived, cells was significantly higher in liver compared with lung, brain $(* p=0.01)$ and spleen $(* * p=0.001)$ in mice eight weeks after induction of endometriosis. Results are expressed as mean \pm SEM. 
particularly interesting as endometriosis is associated with various metabolic abnormalities, including low BMI and metabolic hormone dysregulation [28, 46]. Hepatic involvement may be a precursor of metabolic alterations. The presence of micrometastasis to the liver may help in understanding some of the various systemic metabolic manifestations in endometriosis. Involvement of endometriosis in the Central Nervous System (CNS) has presented as catamenial headache, seizure, papilledema, subarachnoid hemorrhage, and gait disturbance $[17,47]$. Women with endometriosis are more likely to suffer from migraines [48]. There is no classic imaging finding that leads to the diagnosis of endometriosis in the CNS making the true incidence impossible to discern. Similarly, the hypothalamus is the "biological clock" of the neuroendocrine system. It influences appetite,

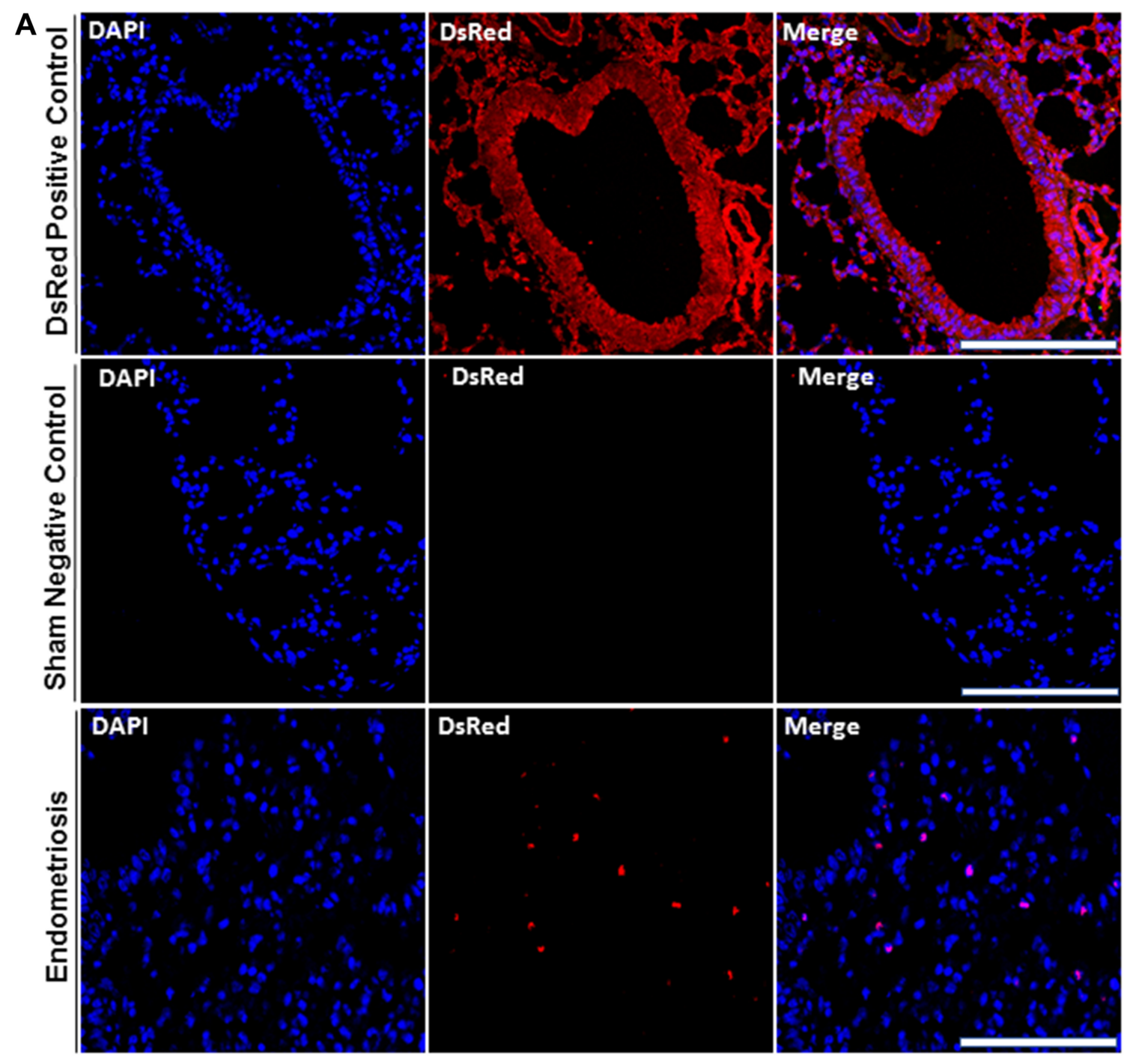

B

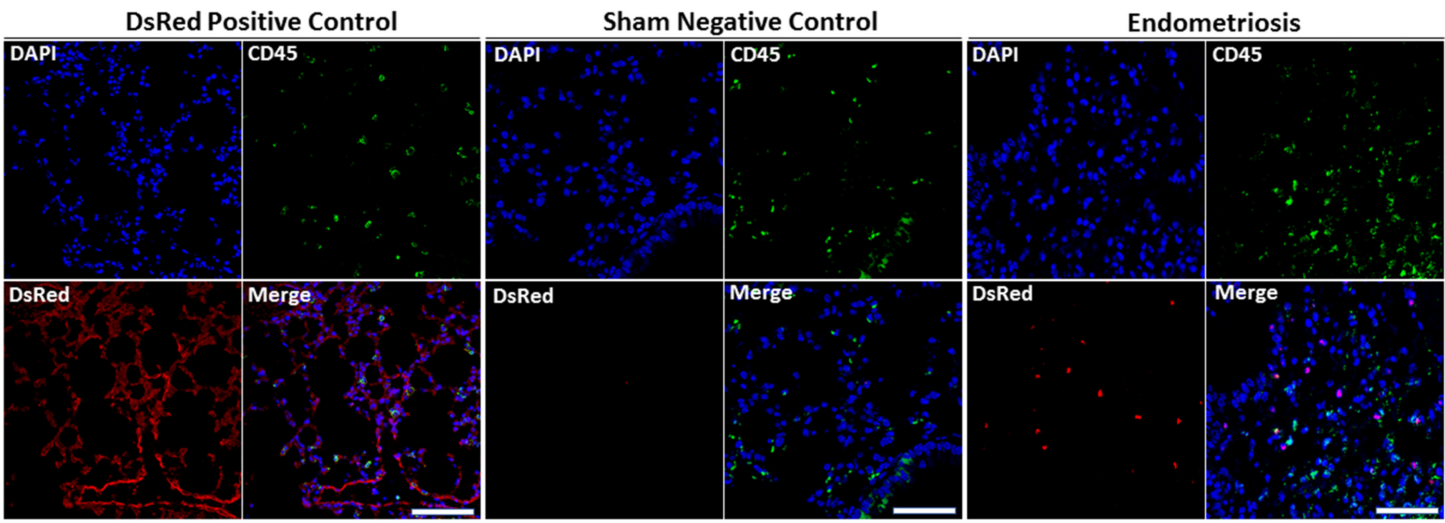


Figure 3: (A) DsRed ${ }^{+}$cells are present in lung parenchyma of mice with endometriosis. Analysis of DsRed ${ }^{+}$cell localization by confocal microscopy showing intense DsRed immunofluorescence in transgenic DsRed mice. No DsRed expression was observed in sham control mice. In each image, blue represents cell nuclei [4', 6'- diamino-2-phenylindole (DAPI)], red indicates DsRed. Scale bars, $100 \mu \mathrm{m}$ (Transgenic DsRed mouse and Sham), $50 \mu \mathrm{m}$ (Endometriosis). Immunofluorescent images are representative of 3 random fields in each slide, with $n=10$ mice in each group, in two independent experiments. (B) DsRed ${ }^{+}$cells did not express CD45 in lung parenchyma of mice following induction of endometriosis by using double immunofluorescence. Confocal microscopic view of lung parenchyma. DsRed ${ }^{+} / \mathrm{Cd}^{4} 5^{-}$cells were observed in the lung tissue of mice with experimental endometriosis. CD45 was used as a leukocyte marker to exclude migrating white blood cells. CD45 cells (leukocytes) were observed, as expected, in all models including controls. Blue staining demonstrates cell nuclei [4', 6'- diamino-2-phenylindole (DAPI)], while red staining corresponds to DsRed, and green staining shows CD45. Scale bars, $100 \mu \mathrm{m}$ (Transgenic DsRed mouse and Sham), $50 \mu \mathrm{m}$ (Endometriosis). The negative control tissues have no DsRed cells but have CD45 leukocytes. The positive control DsRed tissues have all DsRed ${ }^{+}$cells, including the $\mathrm{CD}_{4} 5^{+}$double positive leukocytes. The experimental mice have DsRed ${ }^{+}$cells from the endometriosis that are not leukocytes and are therefore CD45-. Images are representative of three random fields in each slide, with $n=10$ mice in each group, in two independent experiments.

sleep and pituitary gland action, among other functions $[49,50]$. The hypothalamus-pituitary axis has been reported to be compromised in endometriosis, however the underlying mechanisms are unclear [51]. An association between endometriosis and mood disorders has also been previously demonstrated [52]. Women with endometriosis have a higher risk of depression and anxiety than control subjects [53]. Finally, dysregulation of pain perception is also believed to be common in endometriosis [54]. Perhaps some of these clinical manifestations may be due to undetected endometriosis in the CNS. During the short time course of these experiments we did not detect any physiologic manifestations. In humans we suspect that the much longer duration of accumulation may lead to clinical manifestations. Endometriosis has many related systemic symptoms that are currently poorly explained. Some may be due to clinically unrecognized endometriosis micrometastasis.

In Summary, we observed that endometrial-derived cells migrated to extrapelvic organs. Our findings suggest that endometriosis in locations distant from the pelvis may be more common than previously recognized. Endometriosis should be considered a systemic disease that is often subclinical. Single cells or small implants of endometriosis likely may go unrecognized clinically. Our findings may explain the diffuse symptoms that are often considered components of endometriosis. Migration of endometriosis-derived cells to distant organs suggests a contribution of these cells to a wide range of previously unexplained clinical manifestations, including refractory pain, appetite and eating behaviors, pain perception, altered metabolism, and immune dysfunction. Blocking of cellular migration from ectopic lesions to distant areas may be a therapeutic target. Further investigation is necessary to characterize these migratory cells and the potential mechanism of endometriosis-derived cells metastasis in endometriosis.

\section{MATERIALS AND METHODS}

\section{Animal care}

Eight weeks old female transgenic DsRed (Discosoma sp. Red Fluorescent Protein) mice (20) from Jackson Laboratories (Bar Harbor, ME, USA) and female C57BL/6 mice (20) were kept under controlled conditions (a 12-hour light, 12-hour dark cycle, at $22^{\circ} \mathrm{C}$ ) with free access to water and chew. Animals were treated in accordance with a protocol approved by the Yale University Institutional Animal Care and Use Committee (IACUC), and the U.S. Principles for Utilization and Care of Vertebrate Animals Used in Testing, Research, and Training were followed. Briefly, the female transgenic DsRed mice were collected from litters of the same age from different dams of C57BL/6 background. Both parents have construct containing a Red Fluorescent Protein gene (DsRed.MST) under the control of a chicken beta actin promoter.

\section{Murine experimental model of endometriosis}

Twenty C57BL/6 mice were randomly assigned to one of two groups, endometriosis group $(n=10)$ or sham control group $(n=10)$. Experimental endometriosis was created in ten female C57BL/6 mice with intact ovaries as previously described [55]. Briefly, two pieces (7 millimeters) of uterine horn were obtained from transgenic DsRed donor mice. The tissue pieces were split longitudinally to expose the lumen and implanted into the pelvic peritoneum of recipients, bilaterally. Simultaneously, female C57BL/6 mice underwent sham surgery by leaving only suture material in a similar location. Matched DsRed mice were used as a positive control $(n=10)$.

\section{Tissue collection and assessment}

Eight weeks after creation of experimental endometriosis, animals were sacrificed and multiple organs including lung, spleen, liver, and brain were harvested. Fresh tissues were assessed by Fluorescence Activated Cell Sorting (FACS). The remaining tissue was stored in $4 \%$ paraformaldehyde overnight, transferred to $70 \%$ ethanol, and then embedded in paraffin for immunofluorescent analysis.

\section{Fluorescence-activated cell sorting (FACS)}

Lung, spleen, liver, and brain tissue were assessed to determine the presence of DsRed ${ }^{+}$cells using FACS. 


\section{A DsRed Positive Control}

Sham Negative Control

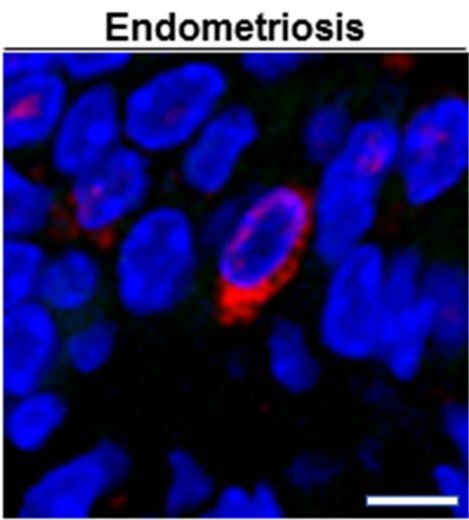

B
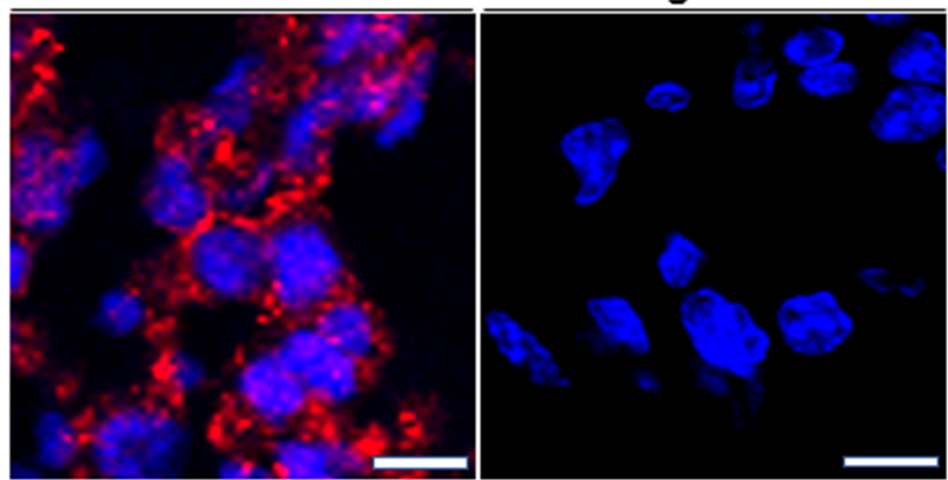

dometriosis

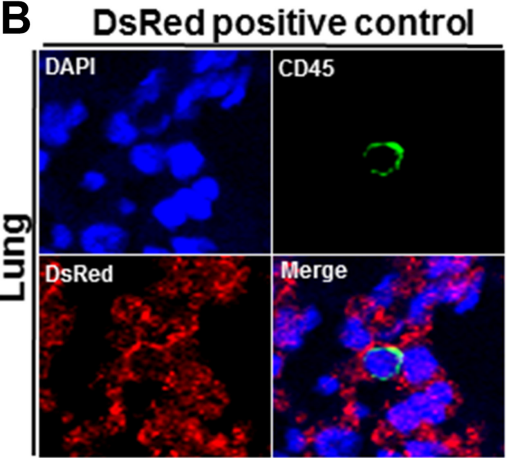

Sham negative control
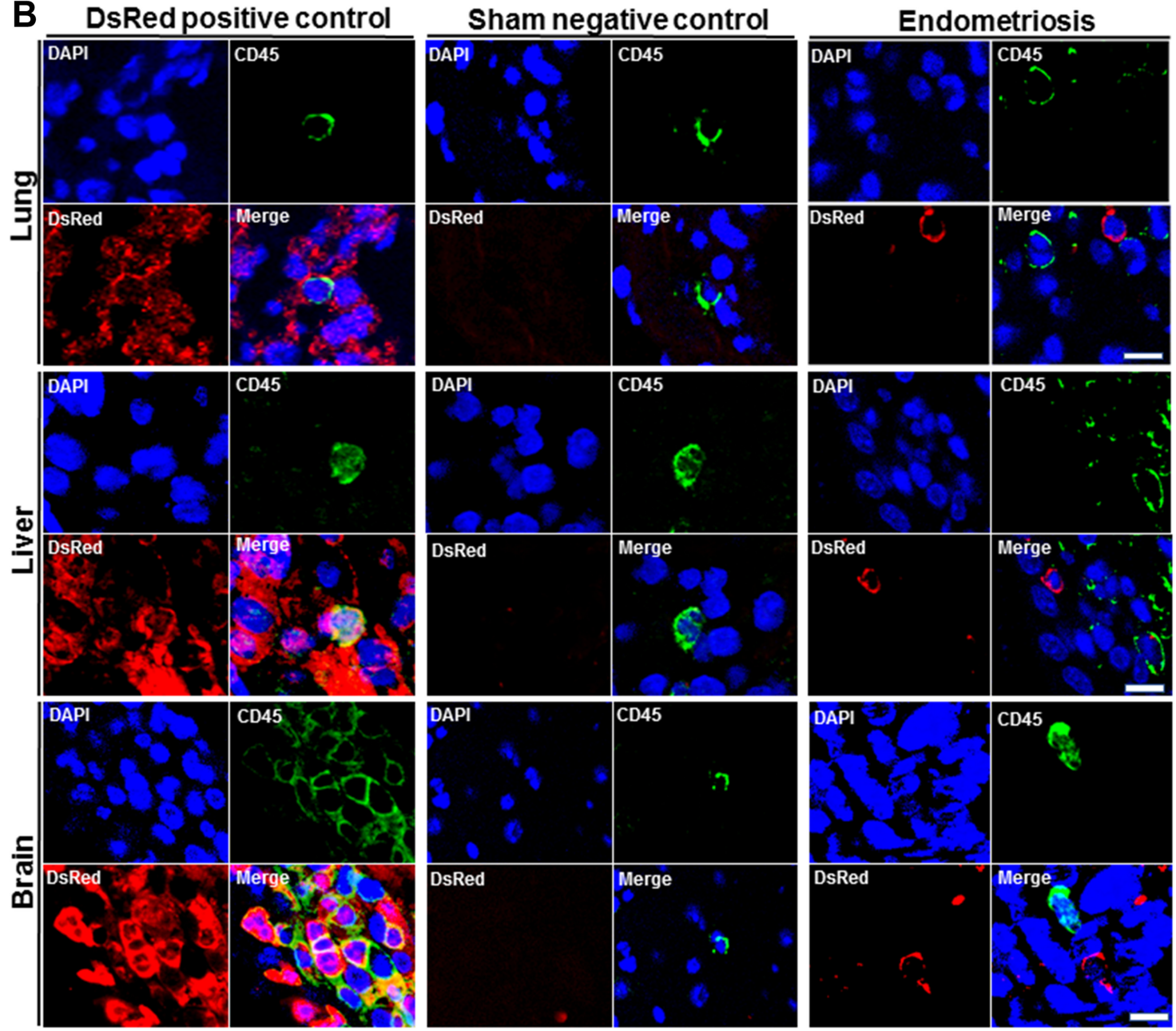

Figure 4: (A) High power immunofluorescence confocal images of DsRed expression in lung, liver, and brain. Representative confocal immunofluorescent images were obtained from lung, liver and brain of all animals after staining with DsRed (Red) and nuclei (blue) (left to right): Transgenic DsRed mouse (positive control), Sham (negative control), Experimental endometriosis model. Scale bars, Lung: $5 \mu$ m (Transgenic DsRed mouse and Endometriosis), $10 \mu \mathrm{m}$ (Sham); Liver: $2.5 \mu \mathrm{m}$ (Transgenic DsRed mouse and Endometriosis), $5 \mu \mathrm{m}$ (Sham); Brain: $4 \mu \mathrm{m}$ (Transgenic DsRed mouse and Endometriosis), $8 \mu \mathrm{m}$ (Sham). Images are representative of three random fields in each slide, with $n=10$ mice in each group, in two independent experiments. (B) Double immunofluorescence (DsRed/CD45) analysis of extrapelvic organs. Analysis by immunofluorescence of lung, liver and brain sections from all animals. DsRed ${ }^{+}$cells did not express CD45 in the extrapelvic organs of mice following induction of endometriosis. CD45 was used as a leukocyte marker to exclude migrating white blood cells. DsRed ${ }^{-} / \mathrm{CD} 45^{+}$cells (leukocytes) were observed in transgenic DsRed and sham model mice. Blue staining demonstrates cell nuclei [4', 6'- diamino-2-phenylindole (DAPI)], while red staining corresponds to DsRed, and green staining shows CD45 positivity. Scale bars, Lung: $5 \mu \mathrm{m}$ (Transgenic DsRed mouse and Endometriosis), $10 \mu \mathrm{m}$ (Sham); Liver: $5 \mu \mathrm{m}$, Brain: $10 \mu \mathrm{m}$. Images are representative of 3 random fields in each slide, with $n=10$ mice in each group, in two independent experiments. 
The specimens were finely minced with surgical scalpels, and subsequently digested with a solution of Hank's Balanced Salt Solution (Life Technologies, Inc, Invitrogen, Carlsbad, CA, USA) containing HEPES (25 mM), collagenase B $(1 \mathrm{mg} / \mathrm{ml}$, Roche Diagnostics, Indianapolis, IN, USA), and deoxyribonuclease (0.1 $\mathrm{mg} / \mathrm{ml}$, Sigma-Aldrich, St. Louis, MO, USA) for $60-90$ min at $37^{\circ} \mathrm{C}$. All samples were filtered using $70-\mu \mathrm{m}$ cell strainers and centrifuged at $3,000 \mathrm{rpm}$ at $4^{\circ} \mathrm{C}$ for 5 minutes. Cell pellets were suspended in FACS buffer and then sorted via fluorescence activated cell sorting (FACS) on a Beckman Coulter MoFlo machine (Beckman Coulter, San Jose, CA, USA). Acquired data were analyzed using the FlowJo V10 software (Tree Star, Ashland, OR, USA) for three independent experiments.

\section{Paraffin-embedded tissue immunostaining}

Paraformaldehyde-fixed specimens were paraffin embedded, cut into $3-\mu \mathrm{m}$ sections, mounted on glass slides, and stained for DsRed and CD45, a hematopoietic cell marker mainly found on leukocytes. Six sections (one/slide) were prepared from each organ of interest of every animal. Three random regions from each organ of the lung, liver and brain were selected in two independent experiments. Sections were deparaffinized and rehydrated through a series of 15-minute xylene and 25-minute ethanol washes and rinsed in Phosphate-Buffered Saline (PBS). The slides with sections were then placed in boiling sodium nitrate $(\mathrm{pH}=6)$ for $10 \mathrm{~min}$ and then treated for 15 min with ammonium chloride in PBS to protect against auto-fluorescence. Sections were incubated in blocking buffer using 5\% donkey serum (Invitrogen) for $30 \mathrm{~min}$ at room temperature followed by primary antibodies: polyclonal goat anti-DsRed IgG antibody (Santa Cruz Biotechnology, Dallas, TX, USA), and rat anti-CD45 antibody (Abcam, Cambridge, MA, USA) diluted 1/200 in $5 \%$ donkey serum and kept in a humidified chamber overnight at $4^{\circ} \mathrm{C}$. Then, the sections were rinsed three times for 20 min respectively in PBS on an orbital shaker and incubated with the following secondary antibodies: donkey anti-goat Alexa Fluor 568 (Life Technologies, Grand Island, NY, USA), and donkey anti-rabbit Alexa Fluor 488 (Life Technologies, Grand Island, NY, USA) diluted 1/500 in PBS for one hr, away from light, at room temperature. Sections were mounted with Vectashield mounting medium with DAPI (4'6-diamidino-2phenylindole, Vector Laboratories, Burlingame, CA, USA) for $15 \mathrm{~min}$ and immunofluorescence signals were observed using a laser scanning confocal microscope (LSM 710, Zeiss, New York, NY, USA). A minimum of 100 highpower microscopic fields were analyzed from triplicate slides in each sample, in three independent experiments. The investigators who performed the tissue investigations were blinded to the experimental groups.

\section{Statistical analysis}

Data were analyzed using GraphPad Prism 5 software (Graph-Pad Software, San Diego, CA, USA) and are presented as the Mean \pm SEM from at least three independent experiments. One-sided analysis of variance (ANOVA) was used to analyze differences in multiple organs comparisons, followed by Tukey's post hoc test. Values were considered to be statistically significantly different if $p<0.05$.

\section{Author contributions}

Dr. Hugh S. Taylor : Obtained funding, designed experiment, assisted with data interpretation and edited manuscript; Dr. Graciela Krikun : Assisted with experimental design and data interpretation; Demetra Hufnagal : Assisted with data interpretation statistical analysis and figure preparation; Levent Mutlu : Assisted with immunofluorescence studies; Fei Li : Assisted with surgical procedures in mice and IHC; Dr. Ramanaiah Mamillapalli : Supervised and assisted with $\backslash$ the entire project, edited manuscript and figures; Elham Samani : Carried out all experiments and initial preparation of manuscript.

\section{CONFLICTS OF INTEREST}

There are no conflicts of interest to disclose.

\section{FUNDING}

This work was supported by National Institutes of Health (R01HD076422).

\section{REFERENCES}

1. Simoens S, Dunselman G, Dirksen C, Hummelshoj L, Bokor A, Brandes I, Brodszky V, Canis M, Colombo GL, DeLeire T, Falcone T, Graham B, Halis G, et al. The burden of endometriosis: costs and quality of life of women with endometriosis and treated in referral centres. Hum Reprod. 2012; 27:1292-9.

2. Bulun SE. Endometriosis. N Engl J Med. 2009; 360:268-279.

3. Giudice LC. Clinical practice. Endometriosis. N Engl J Med. 2010; 362:2389-98.

4. Eskenazi B, Warner ML. Epidemiology of endometriosis. Obstet Gynecol Clin North Am. 1997; 24:235-258.

5. Ozkan S, Murk W, Arici A. Endometriosis and infertility: epidemiology and evidence-based treatments. Ann N Y Acad Sci. 2008; 1127:92-100.

6. Moradi M, Parker M, Sneddon A, Lopez V, Ellwood D. Impact of endometriosis on women's lives: a qualitative study. BMC Womens Health. 2014; 14:1-12. 
7. Sasson IE, Taylor HS. Stem cells and the pathogenesis of endometriosis. Ann N Y Acad Sci. 2008; 1127:106-115.

8. Vercellini P, Vigano P, Somigliana E, Fedele L. Endometriosis: pathogenesis and treatment. Nat Rev Endocrinol. 2014; 10:261-275.

9. Sampson JA. Metastatic or Embolic Endometriosis, due to the Menstrual Dissemination of Endometrial Tissue into the Venous Circulation. Am J Pathol. 1927; 3:93-110.43.

10. Troncon JK, Zani AC, Vieira AD, Poli-Neto OB, Nogueira AA, Rosa-E-Silva JC. Endometriosis in a patient with mayer-rokitansky-küster-hauser syndrome. Case Rep Obstet Gynecol. 2014; 2014:376231.

11. Simsek G, Bulus H, Tas A, Koklu S, Yilmaz SB, Coskun A. An unusual cause of inguinal hernia in a male patient: endometriosis. Gut Liver. 2012; 6:284-285.

12. Vinatier D, Orazi G, Cosson M, Dufour P. Theories of endometriosis. Eur J Obstet Gynecol Reprod Biol. 2001; 96:21-34.

13. Du H, Taylor HS. Contribution of Bone Marrow-Derived Stem Cells to Endometrium and Endometriosis. Stem Cells. 2007; 25:2082-2086.

14. Sakr S, Naqvi H, Komm B, Taylor HS. Endometriosis impairs bone marrow-derived stem cell recruitment to the uterus whereas bazedoxifene treatment leads to endometriosis regression and improved uterine stem cell engraftment. Endocrinology. 2014; 155:1489-1497.

15. Pluchino N, Taylor HS. Endometriosis and Stem Cell Trafficking. Reprod Sci. 2016; 23:1616-1619.

16. Hufnagel D, Li F, Cosar E, Krikun G, Taylor HS. The Role of Stem Cells in the Etiology and Pathophysiology of Endometriosis. Semin Reprod Med. 2015; 33:333-340.

17. Sarma D, Iyengar P, Marotta TR, terBrugge KG, Gentili F, Halliday W. Cerebellar endometriosis. AJR Am J Roentgenol. 2004; 182:1543-1546.

18. Huang $\mathrm{H}, \mathrm{Li} \mathrm{C}$, Zarogoulidis $\mathrm{P}$, Darwiche $\mathrm{K}$, Machairiotis N, Yang L, Simoff M, Celis E, Zhao T, Zarogoulidis K, Katsikogiannis N, Hohenforst-Schmidt W, Li Q. Endometriosis of the lung: report of a case and literature review. Eur J Med Res. 2013; 18:13.

19. Horton JD, Dezee KJ, Ahnfeldt EP, Wagner M. Abdominal wall endometriosis: a surgeon's perspective and review of 445 cases. Am J Surg. 2008; 196:207-212.

20. Markham SM. Extrapelvic endometriosis. In: Thomas EJ, Rock JA, eds. Modern Approaches to Endometriosis. (Dordrecht: Springer Netherlands). 1991; 151-182.

21. Foster DC, Stern JL, Buscema J, Rock JA, Woodruff JD. Pleural and parenchymal pulmonary endometriosis. Obstet Gynecol. 1981; 58:552-556.

22. Ryu JS, Song ES, Lee KH, Cho JH, Kwak SM, Lee HL. Natural history and therapeutic implications of patients with catamenial hemoptysis. Respir Med. 2007; 101:1032-1036.

23. Yao S, Berkowitz E, Najjar H, Tsaltas J. Pleural endometriosis: An unexpected finding during thoracic surgery. BJOG. 2015; 128-129.
24. Vilos GA, Hollett-Caines J, Abu-Rafea B, Ahmad R, Mazurek MF. Resolution of catamenial epilepsy after goserelin therapy and oophorectomy: case report of presumed cerebral endometriosis. J Minim Invasive Gynecol. 2011; 18:128-130.

25. Alvero AB, Kim D, Lima E, Sumi NJ, Lee JS, Cardenas C, Pitruzzello M, Silasi DA, Buza N, Fahmy T, Mor G. Novel approach for the detection of intraperitoneal micrometastasis using an ovarian cancer mouse model. Sci Rep. 2017; 7:40989.

26. Santamaria X, Massasa EE, Taylor HS. Migration of cells from experimental endometriosis to the uterine endometrium. Endocrinology. 2012; 153:5566-5574.

27. Holoch KJ, Lessey BA. Endometriosis and infertility. Clin Obstet Gynecol. 2010; 53:429-438.

28. Sinaii N, Cleary SD, Ballweg ML, Nieman LK, Stratton P. High rates of autoimmune and endocrine disorders, fibromyalgia, chronic fatigue syndrome and atopic diseases among women with endometriosis: a survey analysis. Hum Reprod. 2002; 17:2715-2724.

29. Bitzer J. Psychosomatic aspects of endometriosis. J Endometr. 2011; 3:166-170.

30. Centini G, Lazzeri L, Dores D, Pianigiani L, Iannone P, Luisi S, Petraglia F, Zupi E. Chronic pelvic pain and quality of life in women with and without endometriosis. J Endometr Pelvic Pain Disord. 2013; 5:27-33.

31. Melis I, Litta P, Nappi L, Agus M, Melis GB, Angioni S. Sexual Function in women with deep endometriosis: correlation with quality of life, intensity of pain, depression, anxiety, and body image. Int J Sex Health. 2015; 27:175-185.

32. Lafay Pillet MC, Schneider A, Borghese B, Santulli P, Souza C, Streuli I, de Ziegler D, Chapron C. Deep infiltrating endometriosis is associated with markedly lower body mass index: a 476 case-control study. Hum Reprod. 2012; 27:265-272.

33. Matarese G, De Placido G, Nikas Y, Alviggi C. Pathogenesis of endometriosis: natural immunity dysfunction or autoimmune disease? Trends Mol Med. 2003; 9:223-228.

34. Burney RO, Giudice LC. Pathogenesis and pathophysiology of endometriosis. Fertil Steril. 2012; 98:511-519.

35. Gustofson RL, Kim N, Liu S, Stratton P. Endometriosis and the appendix: a case series and comprehensive review of the literature. Fertil Steril. 2006; 86:298-303.

36. Victory R, Diamond MP, Johns DA. Villar's nodule: a case report and systematic literature review of endometriosis externa of the umbilicus. J Minim Invasive Gynecol. 2007; 14:23-32.

37. Hwang SM, Lee CW, Lee BS, Park JH. Clinical features of thoracic endometriosis: A single center analysis. Obstet Gynecol Sci. 2015; 58:223-231.

38. Redwine DB. Diaphragmatic endometriosis: diagnosis, surgical management, and long-term results of treatment. Fertil Steril. 2002; 77:288-296. 
39. Fukuoka M, Kurihara M, Haga T, Ebana H, Kataoka H, Mizobuchi T, Tatsumi K. Clinical characteristics of catamenial and non-catamenial thoracic endometriosisrelated pneumothorax. Respirology. 2015; 20:1272-1276.

40. Vijitharan V, Pilapitiya S, Premawardana N, Farah M, Siribaddana S. Young woman with recurrent spontaneous pneumothorax: a case report. AMJ. 2015; 9.

41. Suwatanapongched T, Boonsarngsuk V, Amornputtisathaporn $\mathrm{N}$, Leelachaikul P. Thoracic endometriosis with catamenial haemoptysis and pneumothorax: computed tomography findings and long-term follow-up after danazol treatment. Singapore Med J. 2015; 56:e120-123.

42. Tuech JJ, Rousselet MC, Boyer J, Descamps P, Arnaud JP, Ronceray J. Endometrial cyst of the liver: case report and review. Fertil Steril. 2003; 79:1234-1236.

43. Jeanes AC, Murray D, Davidson B, Hamilton M, Watkinson AF. Case report: hepatic and retro-peritoneal endometriosis presenting as obstructive jaundice with ascites: a case report and review of the literature. Clin Radiol. 2002; 57:226-229.

44. Theodosopoulos T, Yiallourou AI, Hatzipappas J, Koutoulidis V, Dadnios N, Contis J. Right-Shoulder Pain: An Unusual Sign of Hepatic Endometriosis. J Gynecol Surg. 2014; 30:383-385.

45. Asran M, Rashid A, Szklaruk J. Hepatic endometriosis mimicking metastatic disease: a case report and review of the literature. J Radiol Case Rep. 2010; 4:26-31.

46. Goetz LG, Mamillapalli R, Taylor HS. Low Body Mass Index in Endometriosis Is Promoted by Hepatic Metabolic Gene Dysregulation in Mice. Biol Reprod. 2016; 95:115.

47. Ichida M, Gomi A, Hiranouchi N, Fujimoto K, Suzuki K, Yoshida M, Nokubi M, Masuzawa T. A case of cerebral endometriosis causing catamenial epilepsy. Neurology. 1993; 43:2708-2709.
48. Yang MH, Wang PH, Wang SJ, Sun WZ, Oyang YJ, Fuh JL. Women with endometriosis are more likely to suffer from migraines: a population-based study. PLoS One. 2012; 7:e33941.

49. Pelluru D, Konadhode RR, Bhat NR, Shiromani PJ. Optogenetic stimulation of astrocytes in the posterior hypothalamus increases sleep at night in $\mathrm{C} 57 \mathrm{BL} / 6 \mathrm{~J}$ mice. Eur J Neurosci. 2016; 43:1298-1306.

50. Di Cristoforo A, Cerri M, Del Vecchio F, Hitrec T, Luppi M, Perez E, Zamboni G, Amici R. Wake-sleep, thermoregulatory, and autonomic effects of cholinergic activation of the lateral hypothalamus in the rat: a pilot study. Arch Ital Biol. 2015; 153:67-76.

51. Quiñones M, Urrutia R, Torres-Reverón A, Vincent K, Flores I. Anxiety, coping skills and hypothalamus-pituitaryadrenal (HPA) axis in patients with endometriosis. J Reprod Biol Health. 2015; 3.

52. Pope CJ, Sharma V, Sharma S, Mazmanian D. A Systematic Review of the Association Between Psychiatric Disturbances and Endometriosis. J Obstet Gynaecol Can. 2015; 37:1006-1015.

53. Chen LC, Hsu JW, Huang KL, Bai YM, Su TP, Li CT, Yang AC, Chang WH, Chen TJ, Tsai SJ, Chen MH. Risk of developing major depression and anxiety disorders among women with endometriosis: A longitudinal follow-up study. J Affect Disord. 2016; 190:282-285.

54. Brawn J, Morotti M, Zondervan KT, Becker CM, Vincent K. Central changes associated with chronic pelvic pain and endometriosis. Hum Reprod Update. 2014; 20:737-747.

55. Naqvi H, Sakr S, Presti T, Krikun G, Komm B, Taylor HS. Treatment with bazedoxifene and conjugated estrogens results in regression of endometriosis in a murine model. Biol Reprod. 2014; 90:121. 